\title{
DYNAMIQUE DE LA POPULATION \\ DE RHIPICEPHALUS PUSILLUS GIL COLLADO, 1938 \\ (ACARINA, IXODIDAE) \\ EN 1986-1987 DANS LE SUD-EST DE L'ESPAGNE : MODÉLISATION DU CYCLE BIOLOGIQUE
}

\author{
F. J. MARQUEZ
}

RÉSUMÉ. L'évolution de la population de Rhipicephalus pusillus, parasite du lapin de garenne. Oryctolagus cuniculus (L.), est étudiée dans une localité de la Province de Granada (Espagne), en 1986 et 1987.

Nous avons étudié, pour chacune des stases du cycle postembryonnaire, les résultats obtenus après application de l'analyse de l'extraction de la tendance aux 358 hôtes considérés. Ceci nous a permis en relation avec les statistiques d'usage habituel, de comprendre, d'une façon plus précise, l'évolution des changements quotidiens qui affectent le nombre moyen de tiques sur la population d'hôtes.

La relation entre les changements observés avec les variations des conditions climatiques et la densité des hôtes est discutée. Divers facteurs nous ont permis d'envisager une dépendance étroite du parasitisme des femelles et des larves vis-à-vis des conditions ambiantes qui, d'une certaine façon, déterminent l'apparition et la concentration de ces parasites sur les hôtes.

Une très forte concordance du parasitisme par les tiques mâles et femelles est observée

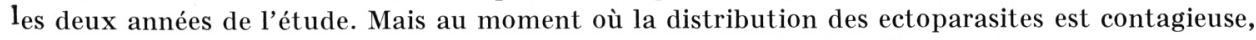
les mâles sont pratiquement deux fois plus abondants que les femelles.

Les larves obéissent à un même type de distribution les deux années, mais il convient de noter un certain décalage, que nous pouvons expliquer par les différences climatiques enregistrées d'une année à l'autre.

Les nymphes, par contre, présentent une courbe de distribution différente les deux années. Leur présence est affectée par les conditions climatiques existantes et surtout par la situation de la population aux moments précédents : ces deux facteurs déterminent un déplacement de leur apparition dans un sens ou dans l'autre.

Mots-clés : Dynamique de population. Moyennes mobiles. Rhipicephalus pusillus (Acarina Ixodidae). Oryctolagus cuniculus (Mammalia, Leporidae).

Evolution of the population of Rhipicephalus pusillus Gil Collado, 1938 (Acarina, Ixodidae) in 1986-1987, in the South East of Spain: modelling of the biological cycle.

SUMMARY. The evolution of the Rhipicephalus pusillus population, a parasite of the european wild rabbit, Oryctolagus cuniculus, is studied in a locality of the Province of Granada (Spain), in 1986-1987.

Inst. "Lopez-Neyra " de Parasitologia. C. S. I. C. Ventanilla, 11, 18001-Granada. Espagne. Accepté le 14 mars 1989. 
The moving average technique was applied to the sampling data (358 hosts), for each stase of the postembrionic cycle. This technique permitted us, together with the habitual usage of the statistics, to understand in a more complete manner, the evolution of daily changes wich affect the average number of ticks over the population of the host rabbits.

Various factors allowed us to envisage a strong dependency of the parasitism of the females and the larvae in relation with the developing environmental conditions, which determine the apparition and concentration of the parasites on the hosts.

A very strong correlation between the parasitism by male ticks and that of the female ticks was observed for the two year duration of our investigation. However, the moment at which the distribution of the ectoparasites is contagious, the male abundance is nearly twice that of the females.

The larvae respond to a similar pattern of distribution both years, but it is necessary to note a delay, which we can attribute to climatic changes registered from one year to the next.

The nymphs, on the other hand, present a different distribution curve for each year. Their presence depends on climatic conditions and especially that of the situation of the ticks and hosts population in the moments proceeding: these conditions determine a deplacement of their apparition in one sense or another.

Key-words: Evolution of the population. Mobile average. Rhipicephalus pusillus (Acarina, Ixodidae). Oryctolagus cuniculus (Mammalia, Leporidae).

\section{Introduction}

L'étude de la biologie des espèces d'Ixodidés parasites de Leporidés (Lepus spp., Silvilagus spp., Oryctolagus cuniculus) a fait l'objet de diverses recherches aux États-Unis et Canada (Campbell et coll., 1980 ; Eddy, 1942 ; Green et coll., 1943 ; McGowan et coll., 1979), en Afrique (Blanc et Bruneau, 1958; Clifford et coll., 1976) et en Europe (Gilot et Rogers, 1979 ; Gilot et coll., 1985; Rodríguez, 1980; Rodríguez et coll., 1979, 1981; Soriguer, 1979, 1980b, 1981b).

Ces études ont été conduites selon un modèle classique qui utilise de manière directe l'information d'échantillonnage. En général, s'effectue une analyse globale des résultats obtenus durant un ou plusieurs cycles annuels, en considérant les valeurs moyennes pour chaque mois ou chaque quinzaine, ou bien la valeur concernant la capture saisonnière d'un certain nombre d'hôtes dans un laps de temps court (Edwards, 1947 ; Gilot et coll., 1975 ; Lees et coll., 1951 ; Main et coll., 1982 ; Mermod et coll., 1973, 1974, 1975 ; Milne, 1947 ; Norval, 1977 ; Piesman et Spielman, 1979 ; Rechav, 1982 ; Schulze et coll., 1986; Wilson et Spielman, 1985 ; et ainsi que les autres auteurs déjà cités).

Les auteurs qui ont travaillé sur Oryctolagus, ont trouvé un spectre de parasitisme par les tiques qui englobe toutes les stases de trois espèces se comportant pratiquement comme parasites stenoxènes de ce Lagomorphe (Haemaphysalis (Rhipistoma) hispanica Gil Collado, 1936, Ixodes (Ixodes) ventalloi Gil Collado, 1936 et Rhipicephalus (Rhipicephalus) pusillus Gil Collado, 1938), en plus des 
stases larvaires de Dermacentor marginatus (Sulzer, 1776) et de Hyalomma lusitanicum Koch, 1844, et ainsi des nymphes de Ixodes ricinus (Linné, 1758).

I. festai Rondelli, 1926 et I. ventalloi ont été longtemps confondus (cf. en particulier Arthur, 1961). Gilot et Perez, 1978 ont montré que leur spécificité est différente et leur détermination est claire. Pour l'identification de $R$. pusillus nous avons utilisé les critères de Morel et Vassiliades, 1962, pour celle de $H$. hispanica nous avons suivi ceux de Hoogstraal et Morel, 1970.

Dans des travaux antérieurs (Márquez et Férnandez-Valdivia, 1987 ; Márquez et Jiménez-Millán, 1988b), nous avons utilisé une approche nouvelle pour les tiques, en appliquant la méthode de l'analyse de la tendance à l'étude de l'évolution de la population des ectoparasites en général, et de certains Ixodidés en particulier (H. hispanica, I. ventalloi, R. pusillus).

Ce travail en est un prolongement des précités. Nous avons, cependant, modifié partiellement la méthode employée (moyennes mobiles sans pondérations), en même temps que nous avons complété notre recherche par une étude analytique de la variance observée.

\section{Matériel et méthodes}

1 - MÉthode de CAPture

Pendant les années 1986 et 1987 nous avons procédé à la récolte des ectoparasites d'une population de lapins de garenne (Oryctolagus cuniculus (L.)), située en Andalousie, à Illora (Granada, Espagne).

Les captures des lapins ont été effectuées au terrier à l'aide de furets (Mustela putorius ssp.), méthode qui a déjà été employée par plusieurs auteurs (Linduska, 1947 ; Soriguer, 1979 ; Gilot et coll., 1985 ; Márquez et Férnandez-Valdivia, 1987 ; Márquez et Jiménez-Millán, 1988b).

Nous avons limité l'échantillonnage à une zone de surface réduite, de presque $10 \mathrm{~km}^{2}$, pour diminuer la variabilité des conditions environnementales qui pourraient conditionner cet échantillonnage.

Dans ce travail, nous présentons les données provenant de 358 lapins adultes et subadultes capturés entre janvier 1986 et décembre 1987 (65 mâles et 103 femelles en 1986, 82 mâles et 108 femelles en 1987). Nous avons retenu exclusivement les résultats provenant d'animaux âgés au minimum de trois mois approximativement : en effet, en adoptant cette limite inférieure d'âge, nous assurons une période minimale de contact entre les lapins de la sous-population étudiée et les populations de parasites qui peuvent potentiellement les affecter. Cette période minimum de contact correspond, en ce qui concerne les lapins d'Andalousie, à la durée d'acquisition d'un poids supérieur à 500 g (Soriguer, 1979, 1980a, 1981b).

Nos données montrent qu'il y a 241 jours pendant lesquels nous avons capturé un ou plusieurs exemplaires (99 jours pendant 1986, ce qui suppose un pourcentage 
de représentation temporelle de $27,1 \%$, et de 142 jours durant 1987, avec une représentation de $38,9 \%$ ). Au total, le pourcentage de représentation temporelle est de $31,6 \%$, au cours des deux années d'étude.

\section{2 - Méthodes statistiQues}

Les résultats obtenus dans les deux échantillonnages annuels ont été étudiés, de façon indépendante, pour les larves, les nymphes, les mâles et les femelles de R. pusillus.

La méthode appliquée a été exposée, pratiquement dans sa totalité, par Márquez et Jiménez-Millán, 1988b, cependant nous avons introduit quelques variantes que nous allons commenter.

Comme dans le travail précité, nous calculons les valeurs moyennes journalières des deux cycles annuels complets, à partir des valeurs réelles observées chez les 358 lapins capturés durant les deux années auxquelles se réfère ce travail.

Nous constatons d'abord qu'il n'y a pas de différence significative entre les données observées dans l'activité parasitaire sur les lapins mâles et femelles. Les données ne correspondent pas à une fonction de distribution normale (test de Kolmogorov-Smirnov). Les moyennes du parasitisme mensuel, saisonnier et annuel, sur les mâles et les femelles, sont comparées par un test de Mann-Withney et nous avons constaté qu'il n'y avait pas de différences significatives entre elles. Ceci nous permet de travailler conjointement sur l'information obtenue sur les deux sexes.

La méthode de calcul change par rapport à celle utilisée par Márquez et Férnandez-Valdivia, 1987 et Márquez et Jiménez-Millán, 1988b, car nous avons mis en évidence un nouvel algorithme qui prend en compte toutes les données impliquées dans le calcul de la valeur moyenne et de la variance pour une date déterminée.

A partir de cette approche, et avec un logiciel que nous avons développé sur le modèle présenté par Legendre et Legendre, 1984, nous avons effectué l'extraction de la tendance, d'après la méthode des moyennes mobiles aux valeurs pondérées.

Le fait d'utiliser cette variante, qui n'a pas été suffisamment cernée par Márquez et Jiménez-Millán, 1988 b, se justifie car, dans l'étude qui nous intéresse ici, nous devons comparer deux nuages de points dans lesquelles les distributions des dates de capture sont complètement différentes. La pondération des données observées nous permet de comparer efficacement les deux distributions pour chacunes des stases impliquées.

La méthode des moyennes mobiles que nous avons employée est fréquemment utilisée pour calculer la tendance dans une série écologique (temporelle ou spatiale). Dans cette méthode itérative, on fait un déplacement, tout le long de la série des données discrètes de capture, des moyennes arithmétiques successives pour $2 \mathrm{~m}$ +1 donnée (Y) contiguë (fenêtre du travail ou amplitude de l'intervalle). Ensuite, nous rapportons la moyenne obtenue $\left(\mathrm{Y}^{\prime}\right)$ à la valeur $\mathrm{m}+1$, centre de la fenêtre considérée. Nous avons pondéré chacune des valeurs des données de capture qui 
sont incorporées dans la fenêtre, en relation inverse à la distance de cette date au point central de la fenêtre, en une relation que finalement on peut exprimer comme :

$$
\mathrm{Y}_{j}^{\prime}=\sum_{i=-m}^{m} \mathrm{Y}_{(j+i)} \mathrm{W}_{i} / \sum_{i=-m}^{m} \mathrm{~W}_{i}
$$

Cette méthode des moyennes itératives est pondéré, avec un facteur $\left(\mathrm{W}_{i}\right)$ qui prend des valeurs entre 1 (pour les données à une distance $m+1$ du centre) et $m+1$ (pour le centre même).

La moitié de l'amplitude de l'intervalle $(m)$, choisi d'après le modèle de la distribution de nos données d'échantillonnage, et qui est déterminée par l'intervalle le plus élevé existant entre deux captures consécutives, est de huit jours.

Choisir la forme du processus (nombre d'itérations) n'est pas simple et dépend de l'objectif que nous avons poursuivi : l'interprétation écologique de la tendance elle-même. Le nombre minimal d'itérations du processus a été fixé expérimentalement à quatre. Le facteur qui marque l'arrêt du processus est en relation directe avec la variance accumulée des moyennes. Celle-ci se réduit à une valeur limite déterminée d'avance, calculée comme la différence entre la variance initiale et finale.

La détermination de la différence entre les valeurs provenant de l'échantillon et les valeurs résultant de l'extraction de la tendance, a été évaluée en comparant l'autocorrélation entre les deux fonctions grâce à un corrélogramme (Legendre et Legendre, 1984).

L'étude de la variance moyenne journalière a été réalisée parallèlement à l'étude de la moyenne journalière du parasitisme, et de la même façon, de sorte que pour les raisons déjà citées, nous pouvons avoir finalement une base de données qui nous permettra de noter le moment et l'importance des changements que l'on constate dans la population de tiques, aussi bien en ce qui concerne les valeurs moyennes observées que la forme dans laquelle ces changements perturbent la distribution de parasites sur leurs hôtes.

Pour utiliser les données climatiques provenant de la station météorologique située dans la zone même d'étude nous avons lissé les données des températures maximale et minimale de la mème façon que nous avons fait pour la moyenne journalière et pour la moyenne de la variance des données.

\section{Résultats}

Les figures 1-4 illustrent la distribution observée des tiques adultes mâles et femelles, des nymphes et des larves de $R$. pusillus au cours des années 19861987. Le tableau $I$ montre les valeurs obtenues par les statistiques de centralisation et dispersion selon les périodes mensuelles. 
Tableau I. - Données mensuelles relatives au parasitisme par les différentes stases de $R$. pusillus $(\mathrm{M}=$ mâle, $\mathrm{F}=$ femelle, $\mathrm{N}=$ nymphe, $\mathrm{L}=$ larve) sur 0 . cuniculus en 1986 (valeur de gauche) et en 1987 (valeur de droite). $n=$ nombre de lapins capturés, D. S. = déviation standard, Min. = minimum, Max. $=$ maximum).

\begin{tabular}{|c|c|c|c|c|c|c|c|c|c|}
\hline \multirow{2}{*}{$\begin{array}{l}\text { Mois } n \\
\text { Janvier }\end{array}$} & \multirow[t]{2}{*}{ Stase } & \multicolumn{2}{|c|}{ Moyenne } & \multicolumn{2}{|c|}{ D. S. } & \multicolumn{2}{|c|}{ Min. } & \multicolumn{2}{|c|}{ Max. } \\
\hline & & & & & & & & & \\
\hline & $\begin{array}{l}\mathrm{M} \\
\mathrm{F}\end{array}$ & $\begin{array}{l}0,58 \\
0,33\end{array}$ & $\begin{array}{l}0,64 \\
0,29\end{array}$ & $\begin{array}{l}0,66 \\
0,65\end{array}$ & $\begin{array}{l}0,74 \\
0,47\end{array}$ & $\begin{array}{l}0 \\
0\end{array}$ & $\begin{array}{l}0 \\
0\end{array}$ & $\begin{array}{l}2 \\
2\end{array}$ & 2 \\
\hline \multirow{2}{*}{$\begin{array}{l}(12) \\
(14)\end{array}$} & $\mathrm{N}$ & 0 & 0,14 & 0 & 0,36 & 0 & 0 & $\begin{array}{l}2 \\
0\end{array}$ & $\begin{array}{l}1 \\
1\end{array}$ \\
\hline & $\mathrm{L}$ & 0 & 0 & 0 & 0 & 0 & 0 & 0 & 0 \\
\hline \multicolumn{10}{|l|}{ Février } \\
\hline & M & 2,38 & 2,08 & 2,06 & 1,50 & 0 & 0 & 6 & 5 \\
\hline \multirow{2}{*}{$\begin{array}{l}(13) \\
(13)\end{array}$} & $\mathrm{F}$ & 1,15 & 1,38 & 0,98 & 1,04 & 0 & 0 & 3 & 3 \\
\hline & $\mathrm{N}$ & $\begin{array}{l}0 \\
0\end{array}$ & $\begin{array}{l}0 \\
0\end{array}$ & $\begin{array}{l}0 \\
0\end{array}$ & $\begin{array}{l}0 \\
0\end{array}$ & $\begin{array}{l}0 \\
0\end{array}$ & $\begin{array}{l}0 \\
0\end{array}$ & $\begin{array}{l}0 \\
0\end{array}$ & $\begin{array}{l}0 \\
0\end{array}$ \\
\hline \multicolumn{10}{|l|}{ Mars } \\
\hline & M & 8,42 & 7,86 & 4,67 & 4,82 & 2 & 2 & 21 & 17 \\
\hline \multirow{3}{*}{$\begin{array}{l}(12) \\
(14)\end{array}$} & $\mathrm{F}$ & 3,42 & 4,43 & 1,88 & 2,79 & 1 & 1 & 7 & 9 \\
\hline & $\mathrm{N}$ & 0 & 0 & 0 & 0 & 0 & 0 & 0 & 0 \\
\hline & $\mathrm{L}$ & 0 & 0 & 0 & 0 & 0 & 0 & 0 & 0 \\
\hline \multicolumn{10}{|l|}{ Avril } \\
\hline & M & 15,13 & 15,73 & 8,03 & 4,17 & 6 & 9 & 32 & 26 \\
\hline \multirow{2}{*}{$\begin{array}{l}(15) \\
(15)\end{array}$} & $\mathrm{F}$ & 6,07 & 6,53 & $\begin{array}{l}2,84 \\
0\end{array}$ & 2,10 & $\begin{array}{l}2 \\
0\end{array}$ & $\begin{array}{l}3 \\
0\end{array}$ & $\begin{array}{r}11 \\
0\end{array}$ & $\begin{array}{r}11 \\
0\end{array}$ \\
\hline & $\mathrm{L}$ & 0 & 0,60 & 0 & 1,24 & 0 & 0 & 0 & 0 \\
\hline \multicolumn{10}{|c|}{$3,93 \quad 5,09$} \\
\hline & M & 12,37 & 13,76 & 3,93 & 5,09 & 7 & 6 & 19 & 23 \\
\hline \multirow{2}{*}{$\begin{array}{l}(16) \\
(17)\end{array}$} & $\mathrm{F}$ & 5,62 & 5,59 & 1,66 & 2,83 & 3 & 1 & 8 & 11 \\
\hline & $\begin{array}{l}\mathrm{N} \\
\mathrm{L}\end{array}$ & 0,25 & $\begin{array}{r}0,59 \\
13,35\end{array}$ & $\begin{array}{l}0 \\
0,77\end{array}$ & $\begin{array}{r}1,28 \\
12,29\end{array}$ & $\begin{array}{l}0 \\
0\end{array}$ & $\begin{array}{l}0 \\
0\end{array}$ & $\begin{array}{l}0 \\
3\end{array}$ & $\begin{array}{l}0 \\
4\end{array}$ \\
\hline \multicolumn{10}{|l|}{ Juin } \\
\hline & M & 16,93 & 9,50 & 3,93 & 4,75 & 4 & 3 & 50 & 21 \\
\hline \multirow{3}{*}{$\begin{array}{l}(15) \\
(16)\end{array}$} & $\mathrm{F}$ & 5,62 & 3,56 & 1,66 & 2,31 & 0 & 1 & 28 & 9 \\
\hline & $\mathbf{N}$ & 19,80 & 11,37 & 32,66 & 9,70 & 0 & 0 & 96 & 31 \\
\hline & $\mathrm{L}$ & 124,40 & 70,19 & 57,17 & 37,74 & 26 & 17 & 185 & 142 \\
\hline \multicolumn{10}{|l|}{ Juillet } \\
\hline \multirow{3}{*}{$\begin{array}{l}(14) \\
(17)\end{array}$} & $\underset{\mathrm{F}}{\mathrm{M}}$ & $\begin{array}{l}5,07 \\
2,14\end{array}$ & $\begin{array}{l}3,06 \\
1,47\end{array}$ & $\begin{array}{l}2,55 \\
1,03\end{array}$ & $\begin{array}{l}1,15 \\
0,87\end{array}$ & $\begin{array}{l}1 \\
0\end{array}$ & $\begin{array}{l}1 \\
0\end{array}$ & $\begin{array}{r}11 \\
4\end{array}$ & 3 \\
\hline & $\mathrm{N}$ & 84,00 & 44,23 & 32,79 & 29,86 & 17 & 12 & 132 & 117 \\
\hline & $\mathrm{L}$ & 107,00 & 112,65 & 24,58 & 50,60 & 78 & 25 & 163 & 210 \\
\hline \multicolumn{10}{|l|}{ Août } \\
\hline & M & 3,53 & 1,69 & 1,41 & 1,14 & 2 & 0 & 7 & 4 \\
\hline $\begin{array}{l}(15) \\
(16)\end{array}$ & $\mathrm{F}$ & 1,73 & 0,81 & $\begin{array}{r}1,39 \\
5157\end{array}$ & $\begin{array}{r}0,75 \\
2004\end{array}$ & $\begin{array}{r}0 \\
39\end{array}$ & $\begin{array}{r}0 \\
63\end{array}$ & $\begin{array}{r}5 \\
231\end{array}$ & 132 \\
\hline (10) & $\mathrm{L}$ & $\begin{array}{r}104,80 \\
59,73\end{array}$ & $\begin{array}{l}81,12 \\
83,12\end{array}$ & $\begin{array}{l}51,01 \\
35,01\end{array}$ & $\begin{array}{l}20,04 \\
32,13\end{array}$ & 16 & 37 & 132 & 143 \\
\hline
\end{tabular}


Tableau I (suite).

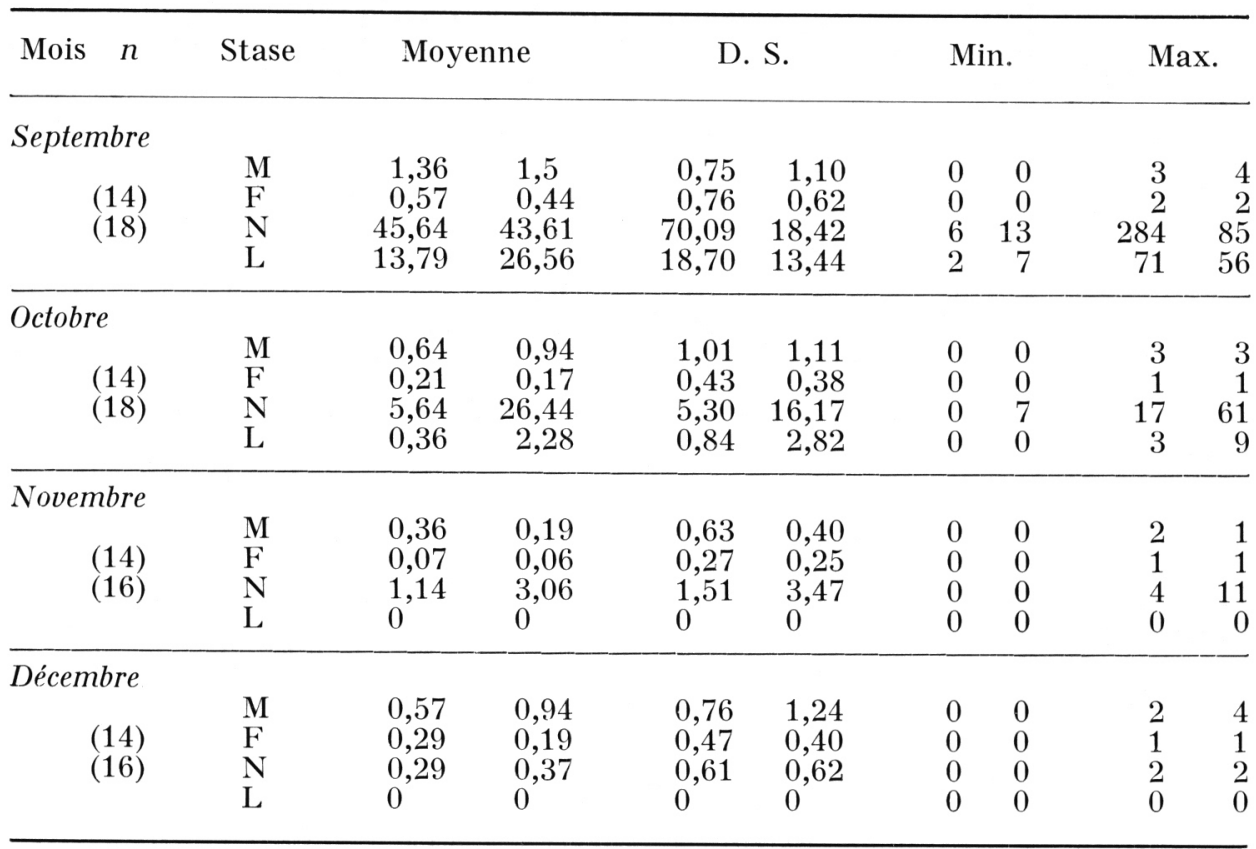

Pendant ces deux années, on peut observer un maximum printanier dans la distribution des adultes (fig. 1 pour les mâles, fig. 2 pour les femelles). En général, nous avons trouvé sur chaque hôte, un nombre plus élevé de mâles que de femelles.

Quant aux larves (fig. 3), on remarque un certain décalage dans leur apparition d'une année sur l'autre. En 1987, les larves sont apparues sur les hôtes un peu plus tôt, mais nous pouvons remarquer que leur maximum se situe presque un mois et demi après le maximum observé en 1986. Cette différence sera commentée un peu plus loin.

Pour ce qui concerne les nymphes (fig. 4), elles apparaissent pendant la période de mai à juin (un petit peu en retard en 1987), avec une période de décalage d'environ un mois par rapport au moment où les premières larves font leur apparition.

Nous présentons dans les figures 5, 6, 7 et 8 l'ajustement de la distribution des valeurs du parasitisme moyen journalier (ligne continue) et de la variance moyenne journalière (aire sombre), pour chacune des stases de $R$. pusillus durant 1986 et 1987.

Mâles.

Nous pouvons observer un comportement identique quant aux périodes d'apparition, de disparition et la valeur maximale atteinte par les mâles de $R$. pusil- 

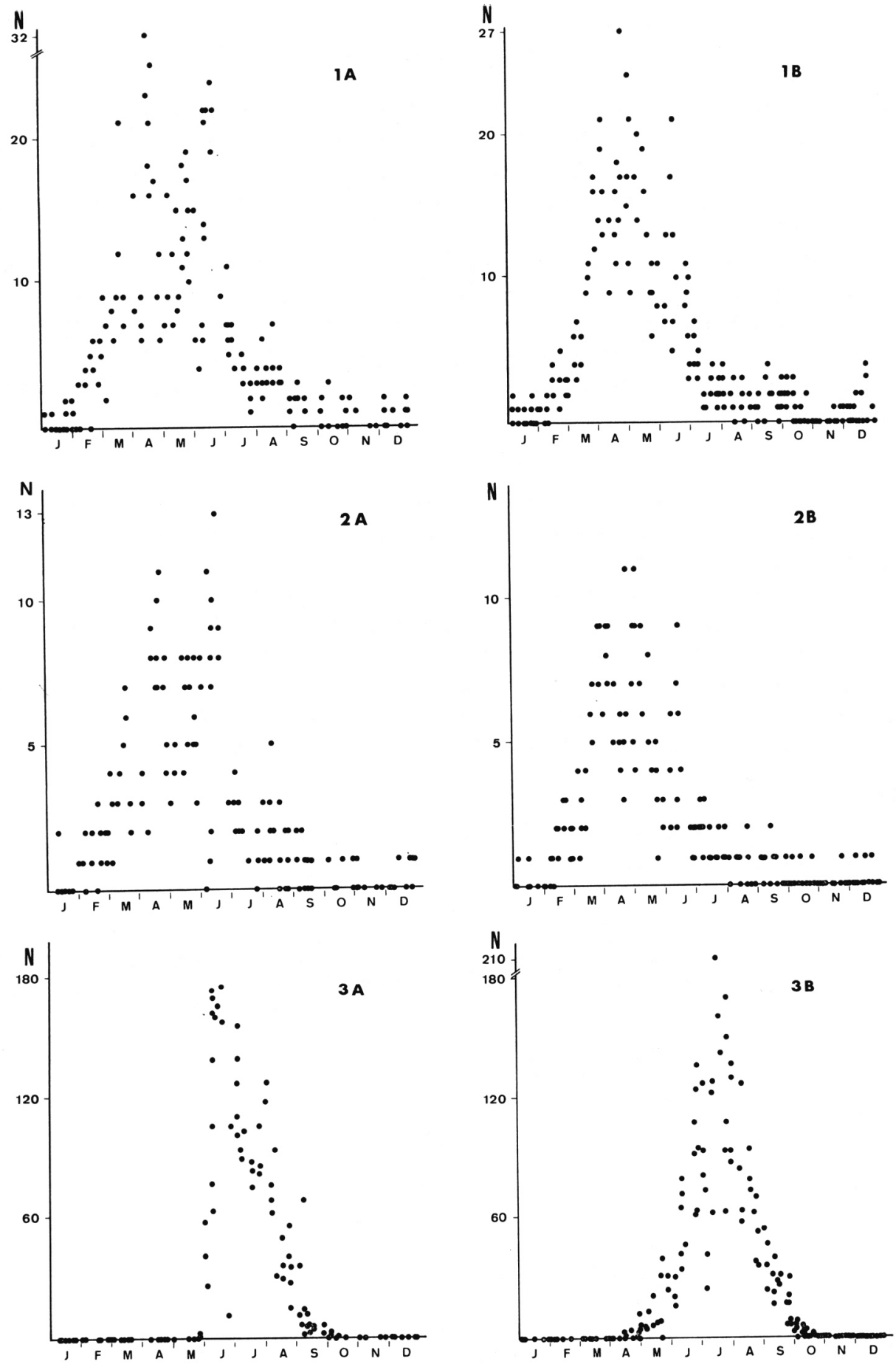

FIG. 1. - Distribution des valeurs du nombre de $R$. pusillus mâle par lapin en 1986 (A) et 1987 (B) à Illora (Granada, Espagne).

Fig. 2. - Distribution des valeurs du nombre de $R$. pusillus femelle par lapin en $1986(\mathrm{~A})$ et 1987 (B) à Illora (Granada, Espagne).

FIG. 3. - Distribution des valeurs du nombre de $R$. pusillus larve par lapin en 1986 (A) et 1987 (B) à Illora (Granada, Espagne). 
lus durant 1986 (fig. 5 A) et 1987 (fig. 5 B); par contre, il faut noter la chute brusque du nombre moyen des individus qui parasitent chaque hôte en avril-mai.

En 1987 les mâles de $R$. pusillus se sont comportés de manière différente de sorte, que le dernier sommet de la courbe, situé début juin, s'est réduit considérablement.

Si l'on considère la variance, nous pouvons remarquer que, pendant 1986, celle-ci a atteint des valeurs particulièrement élevées par rapport à ce que nous avons observé l'année suivante, avec deux maximaux centrés sur les dates lorsque la sous-population de lapins considérée se trouve très fortement parasitée.

Autrement dit, la variance observée durant 1987 ne présente pas ces grandes différences, mais est plutôt nettement stabilisée.

Femelles.

Le cas des femelles est semblable à celui des mâles, avec lequel il garde une forte ressemblance (tableau II). L'évolution observée en 1986 (fig. 6 A) est semblable à celle de 1987 (fig. 6 B).

De son côté, la variance présente la même disposition (tableau III), de sorte que nous pouvons dépister un comportement similaire dans le parasitisme exercé par les mâles et les femelles, pendant la même période.

Les différences numériques entre les deux sexes sont dues au comportement distinct observé, ce qui permet de souligner une adaptation différente en fonction de la finalité du processus d'alimentation de chaque sexe.

\section{Larves.}

Les résultats que nous avons dégagé des larves (fig. 7), sont assez semblables (tableau II) pour les deux années.

Tableau II. - Coefficients de corrélation entre la moyenne journalière, pour les différentes stases de $R$. pusillus $(\mathrm{M}=$ mâle, $\mathrm{F}=$ femelle, $\mathrm{N}=$ nymphe, $\mathrm{L}=$ larve) en 1986 et 1987 . Tous les coefficients sont significatifs à 0,999 sauf $*$ à 0,99 .

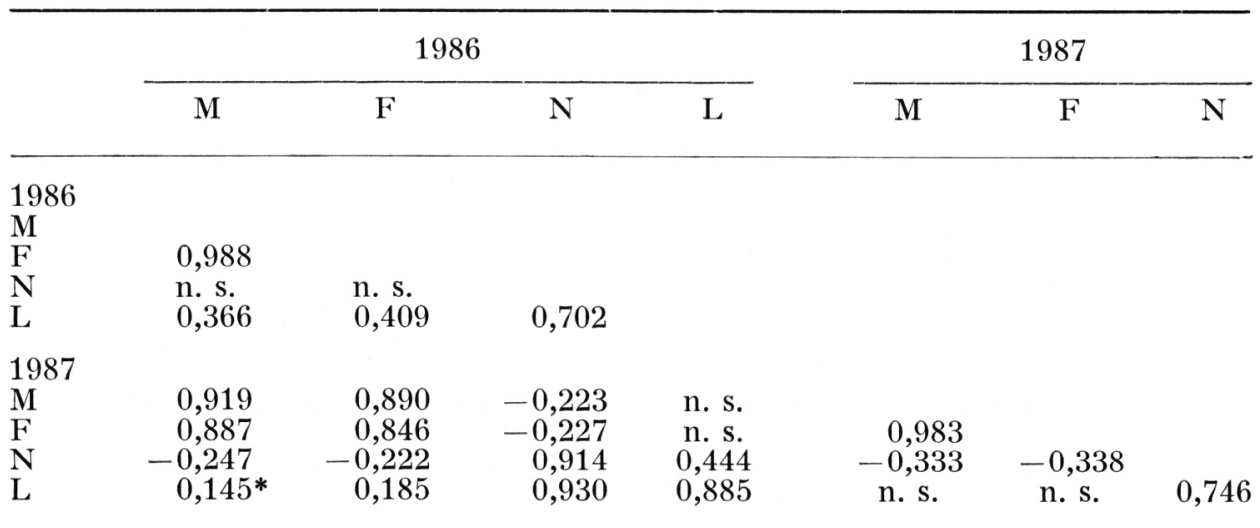



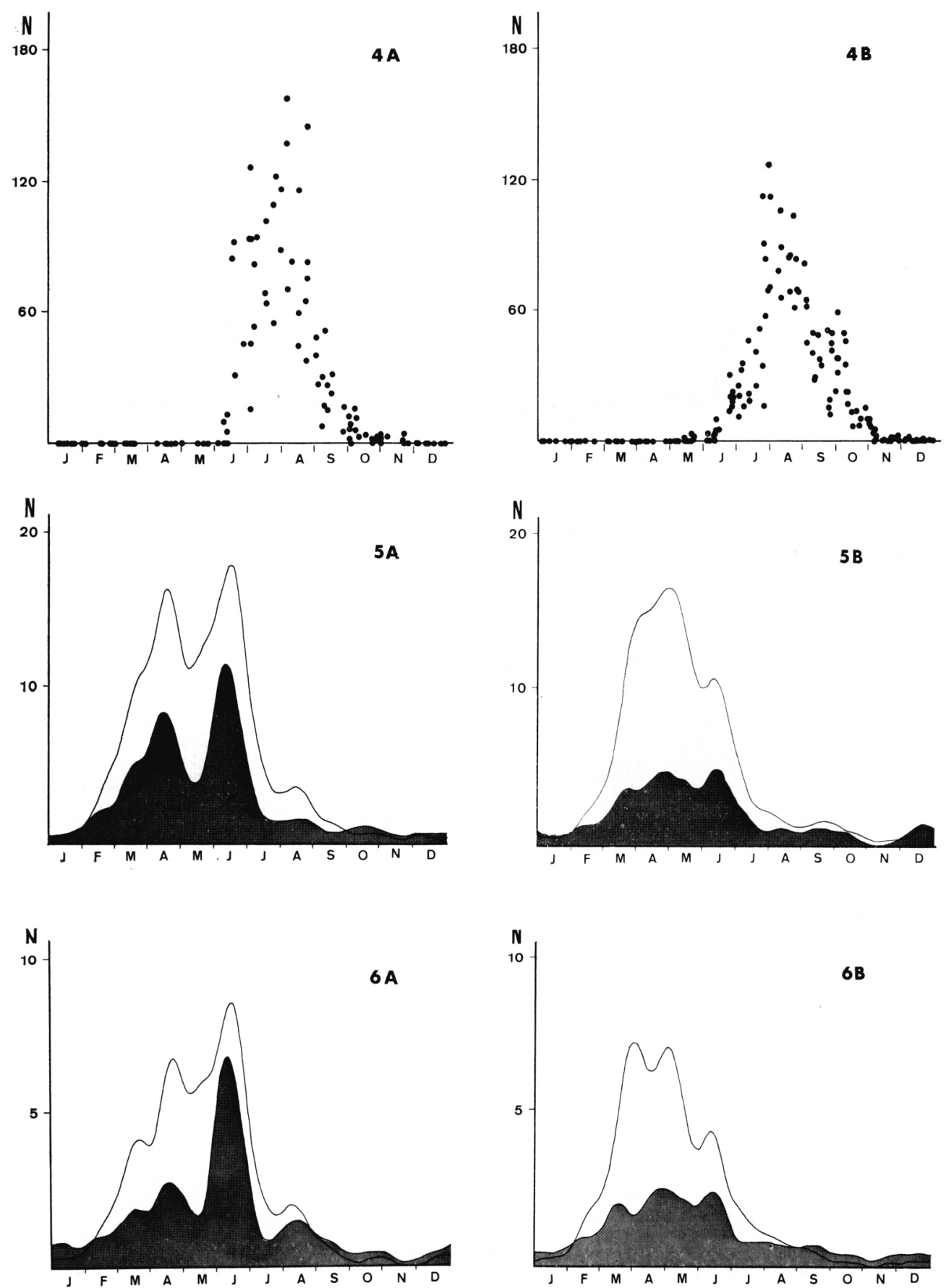

FIG. 4. - Distribution des valeurs du nombre de $R$. pusillus nymphe par lapin en 1986 (A) et 1987 (B) à Illora (Granada, Espagne).

FIg. 5. - Évolution journalière des valeurs de la moyenne (aire claire) et de la variance (aire sombre) de R. pusillus mâle par hôte en 1986 (A) et 1987 (B).

FIG. 6. - Évolution journalière des valeurs de la moyenne (aire claire) et de la variance (aire sombre) de R. pusillus femelle par hôte en 1986 (A) et 1987 (B). 

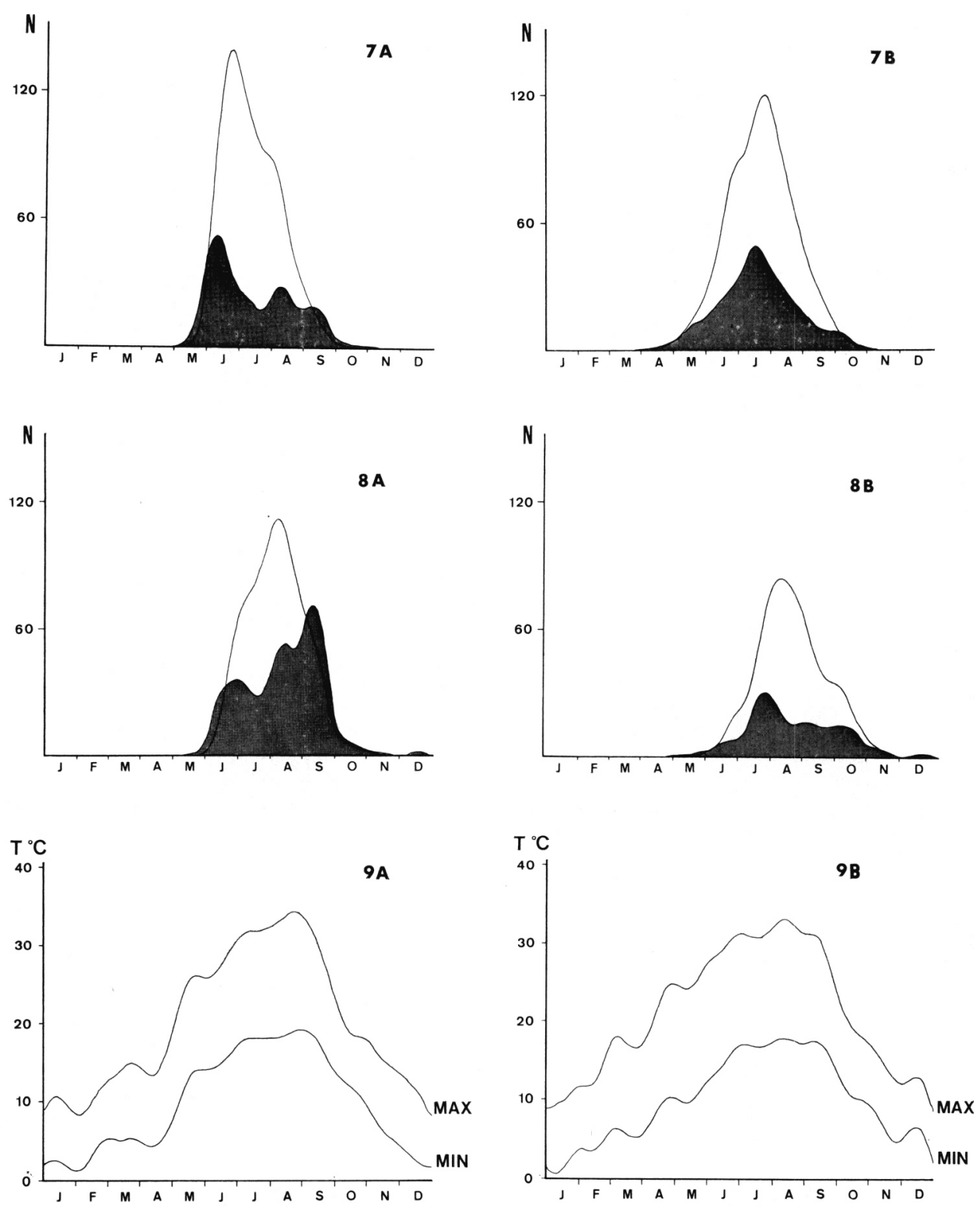

FIG. 7. - Évolution journalière des valeurs de la moyenne (aire claire) et de la variance (aire sombre) de $R$. pusillus larve par hôte en 1986 (A) et 1987 (B).

Fig. 8. - Évolution journalière des valeurs de la moyenne (aire claire) et de la variance (aire sombre) de $R$. pusillus nymphe par hôte en 1986 (A) et 1987 (B).

FIG. 9. - Évolution de la température maximale et minimale journalière moyenne dans l'aire d'étude en 1986 (A) et en 1987 (B). 
TABleau III. - Coefficients de corrélation entre la variance journalière, pour les différentes stases de $R$. pusillus $(\mathrm{M}=$ mâle, $\mathrm{F}=$ femelle, $\mathrm{N}=$ nymphe, $\mathrm{L}=$ larve) en 1986 et 1987 . Tous les coefficients significatifs à 0,999 sauf $*$ à 0,99 .

\begin{tabular}{|c|c|c|c|c|c|c|c|}
\hline & \multicolumn{4}{|c|}{1986} & \multicolumn{3}{|c|}{1987} \\
\hline & M & $\mathrm{F}$ & $\mathrm{N}$ & $\mathrm{L}$ & M & $\mathrm{F}$ & $\mathrm{N}$ \\
\hline \multicolumn{8}{|l|}{$\begin{array}{l}1986 \\
M\end{array}$} \\
\hline $\mathrm{F}$ & 0,930 & & & & & & \\
\hline $\mathrm{N}$ & n. $s$. & 0,114 & & & & & \\
\hline $\mathrm{L}$ & 0,499 & 0,729 & 0,646 & & & & \\
\hline $\begin{array}{l}1987 \\
\text { M }\end{array}$ & 0921 & 0.798 & $-0.143 *$ & 0.329 & & & \\
\hline $\mathrm{F}$ & 0,892 & 0,772 & $-0,136^{*}$ & 0,299 & 0,973 & & \\
\hline $\mathrm{N}$ & $-0,27 \overline{3}$ & $-0,133^{*}$ & 0,760 & 0,461 & $-0,320$ & $-0,314$ & \\
\hline L & 0,168 & 0,317 & 0,671 & 0,758 & n. s. & n. s. & 0,768 \\
\hline
\end{tabular}

Les larves ont une seule période d'apparition, entre les mois de mai à septembre, avec un maximum en juin de 1986 et juillet-août de 1987.

En ce qui concerne les variances, en 1986 on a observé un déplacement vers le début de la période d'apparition, par contre, celle de 1987 est centrée dans cette période.

Nymphes.

L'apparition des nymphes (fig. 8) se produit approximativement un mois après celle des larves, avec le même décalage que celui observé pour celles-ci, mais il semble que ce décalage soit en partie réduit.

Les nymphes de $R$. pusillus apparaissent sur les hôtes entre les mois de mai et de novembre, avec seulement un maximum situé en août.

Il y a une notable différence entre les valeurs maximaux atteintes en 1986 et en 1987, bien que ces valeurs se trouvent aux mêmes dates.

La variance se trouve fortement augmentée pendant la première année d'étude, à la fin de la période d'apparition des nymphes. Par contre, durant la deuxième année, la courbe de la variance a son maximum précisément au commencement de la période d'apparition des nymphes.

\section{Discussion}

La dynamique des populations des mâles, des femelles, des larves et des nymphes de $R$. pusillus, que nous avons étudié au cours de deux cycles annuels, présente une série de caractéristiques qui peuvent être résumées ainsi :

1 - Durant les deux années d'étude, nous avons observé une apparition 
saisonnière presque continue des adultes mâles et femelles, avec une ou deux maximaux centrés au cours des mois d'avril à juin. En ce qui concerne les deux cycles annuels, on remarque la présence pratiquement constante des adultes de l'espèce, bien qu'il faut signaler que, probablement, une partie des individus qui font leur apparition à partir de juillet-août, et avec certitude, ceux qui apparaissent en octobre-décembre des deux années, sont des adultes de deuxième génération, provenant des œufs qui ont éclos cette même année.

Pour les mâles de R. pusillus, pendant les deux années, on peut observer une chute pendant avril-mai dans les courbes des séries de moyennes mobiles, qui est due, en principe, à la dispersion d'une population de tiques montrant une légère hausse dans une population de lapins en pleine expansion, et qui commence à décliner vers la fin du printemps (Soriguer, 1979, 1981a, 1981b).

Dans ce sens, nous croyons que les variations appréciées dans la distribution de la variance moyenne entre les mâles des deux années peuvent s'expliquer grâce à deux facteurs qui sont imbriqués. D’une part, il se produit un déplacement dans le temps des maximaux, causé sûrement par des changements ambiants, influencé essentiellement par la distribution des températures (Aubert, 1975 ; Daniel et coll., 1974, 1980). D'autre part, il y ent une modification comportementale chez les lapins, provoquée par une faible compétitivité spatiale.

Cette modification se traduirait finalement en une réduction de la variabilité des temps de séjour passés par chaque individu dans le terrier, et par conséquent, cela conditionnerait une homogénéisation de la probabilité d'infestation par ces parasites, pratiquement pholéophiles.

2 - Il existe une très grande correspondance entre les résultats obtenus chez les adultes pendant les deux cycles. Les corrélations observées entre mâles et femelles d'une même période (tableau II) sont légèrement plus élevées que celles existantes pour un sexe donné au cours des deux années, aspect qui n'est pratiquement pas noté par Gilot et coll., 1985.

Il y a une graduation entre ces valeurs : les valeurs de corrélation entre mâles et femelles de la même année sont plus fortes que la valeur de corrélation entre les mâles des deux années, qui toutefois, est plus grande que la valeur obtenue chez les femelles. D'autre part, les corrélations croisées entre les mâles d'une année et les femelles de l'autre, offrent des valeurs semblables dans les deux cas, et toujours supérieures aux valeurs de corrélation entre les femelles.

Tout ceci peut être interprété dans le sens signalé antérieurement : le comportement des mâles pendant les deux années est plus indépendant des conditions ambiantes que celui des femelles, et c'est à partir de ce fait que l'on peut exprimer la haute corrélation existant entre les mâles par opposition à celle des femelles. Comme nous l'avons souligné antérieurement (Márquez et Jiménez-Millán, 1988a), les différences numériques observées peuvent être expliquées par le comportement alimentaire différent de chaque sexe.

3 - L'évolution des populations des larves pendant ces deux périodes est très semblable. En observant les figures $\% A$ et $\% B$, on peut constater l'existence 
d'une relation entre l'apparition des larves sur leurs hôtes (période d'activité étroitement relié à l'éclosion des larves) et la température maximale et minimale du mois précédent (Aubert, 1975).

Les différences observées sur les maximal sont en relation éventuelle avec la réduction de la probabilité de fixation sur un hôte, mais surtout dans la période effective de l'apparition des parasites. Nous croyons dans ce cas que les variations observées peuvent être interprétées par les différences climatiques, surtout en ce qui concerne la température (Daniel et coll., 1977, 1980 ; Honzakova et coll., 1980), notées pendant les deux années (fig. 9). De ce fait, la grande pente qui apparaît dans la courbe établie en 1986 peut s'expliquer par l'apparition massive d'un ensemble de larves qui, par une prolongation inégale de leur période de développement embryonnaire devraient avoir terminé leur éclosion de façon presque synchrone (en relation avec l'hypothèse développe par MacLeod, 1936 et Arthur, 1951, confirmé par Randolph, 1975 et modélisé par Arthur, 1975). Par contre, en 1987, on observe une augmentation constante dans l'apparition des larves, donnant lieu à une pente beaucoup plus atténuée dans la courbe des moyennes.

De la même façon, le déplacement relatif des maximaux, est traduit comme une différence provoquée par leur année d'origine (effet de retard de la ponte et de l'éclosion des oufs pendant 1986), mais en même temps ces variations peuvent être considérées comme une possible conséquence des potentialités différentes des populations en 1986 et 1987.

De cette manière, nous pouvons expliquer, d'une façon cohérente, les différences entre les moments d'apparition et les pentes des courbes moyennes journalières des larves pour les deux périodes.

La corrélation modérée observée entre les deux distributions, comme celle que nous avons commenté antérieurement dans le cas des femelles, est due, pensonsnous, surtout aux différences climatiques entre ces deux années.

4 -- Les différences observées entre les nymphes des deux générations, sont en relation, à notre avis, avec les conditions initiales existant au début de chaque année (densité préalable de tiques et d'hôtes, etc.), plus qu'avec les conditions climatiques du moment où elles se développent. La distribution des nymphes et des larves de la même période, semblable dans les deux cas, est peu corrélée, ce qui semble confirmer cette hypothèse.

Cependant, on peut s'étonner de la très grande différence observée entre les corrélations croisées entre nymphes et larves des différentes périodes : très grande pour les nymphes de 1986 et les larves de 1987, très faibles pour les larves de 1986 et les nymphes de 1987. Dans ce cas, nous croyons que ce phénomène est une conséquence de la conjonction d'une série de facteurs. Ces facteurs, fondamentalement marqués par la tendance existant dans la population de tiques, et aussi dans la population de lapins dans le sens d'une réduction des effectifs (arthur, 1975) et par les conditions climatiques qui ont favorisé le décalage temporel des larves et des nymphes, ces facteurs, disons-nous, ont par leur réunion finalement entraîné l'existence d'une haute corrélation, qui en principe n'aurait pas dû s'observer. 
La réduction dans le décalage observé, par rapport à ce des larves peut être un effet semblable à celui de la diffusion d'un signal dans un milieu imparfaitement élastique.

Nous croyons que la variation entre les valeurs maximales est due fondamentalement à la densité différente des hôtes observée durant ces deux années, et que finalement celle-ci se reflète dans la valeur du parasitisme des nymphes sur les lapins, parasitisme également abaissé (Mermod et coll., 1973, 1974, 1975).

L'explication de les différences des tendances des deux variances peut avoir son origine dans le changement de densité des hôtes (reproduction automnale différente et plus grande virulence de la myxomatose pendant 1986) et dans un effet d'accumulation des pertes dans la population de tiques, provenant d'une capacité plus réduite du milieu (réduction de la densité des hôtes au cours d'une grande période de temps correspondant au maintien des causes de mortalité dans la population d'ectoparasites par l'augmentation de la résistance immune de l'hôte (Randolph, 1979 ; McGowan et coll., Willadsen, 1980)), etc.

5 - Nous pouvons observer une très grande concordance de nos résultats avec ceux obtenus par Gilot et coll., 1985 à la Tour du Valat en Camargue (Bouches-du-Rhône, France) pour le parasitisme par les adultes de $R$. pusillus dans les mois hivernaux, mais en raison de la méthodologie employée, ces résultats diffèrent légèrement pendant le printemps et l'été. Les larves et les nymphes, à cause d'une information trop fragmentaire, sont difficilement comparables, toutefois quelles sont trouvées seulement dans l'échantillonnage d'août.

Rodriguez, 1980, et Rodriguez et coll., 1979, 1981, ont effectué leurs recherches dans un endroit proche du nôtre (Ciudad Real, Espagne). Malheureusement, ils ont réuni les données provenants de lapins et de lièvres (Lepus europaeus (L.)), de sorte qu'il est presque impossible de les comparer avec les nôtres. Cette espèce de Lepus citée devrait être assimilée à Lepus granatensis Rosenhauer, 1856, unique espèce de ce genre se trouvant dans cette région d'Espagne (Palacios, 1983).

\section{Conclusions}

L'application de la méthode d'extraction de la tendance, concernant les études de l'évolution des populations de tiques, s'avère de grande utilité puisqu'elle nous permet de considérer un ensemble important d'informations que les méthodes habituellement utilisées ne peuvent cerner de manière efficace.

Il faut considérer que la population de tiques, se trouve insérée dans une dynamique dans laquelle, pour des périodes relativement courtes, sont impliquées aussi bien les conditions climatiques que les variations possibles dans la densité des hôtes.

Dans ce sens, nous croyons que les conditions initiales existant au début de chaque année déterminent, au moins en partie, le développement ultérieur de cette dynamique de la population. 
La variation des conditions climatiques, dans ce domaine d'étude, influe sur les femelles et les larves. La température exerce une pression capitale sur le temps moyen d'alimentation des femelles et la durée de leur ponte, le développement embryonnaire des larves et la mue entre les différentes stases; elle joue un rôle critique lorsqu'elle intervient comme facteur limitant capable de stopper ce processus (hiver et printemps). De ce point de vue, il est aisé de constater l'importance de ces effets sur les femelles et les larves.

\section{RÉFÉRENCES}

Arthur D. R. : The synonymy of Ixodes festai Rondelli, 1926. Parasitology, 1961, 51, 497.

Arthur D. R. : Modelling of ticks populations as a prerequisite to control. British Insect and Fungic Conference, 1975, 1025-1033.

Aubert M. F. A. : Contributions à l'étude des ectoparasites du renard et d'autres Carnivores sauvages de l'Est de la France. Interprétation de la dynamique saisonnière en relation avec la biologie de l'hôte. Acarologia, 1975, 1\%, 452-479.

Blanc G., Bruneau J. : Apparition saisonnière des Ixodes sur lapins, petits mammifères et lézards de la forêt de Nefifik. Archs. Inst. Pasteur Maroc, 1958, 5, 493-498.

Campbell A., Ward R. M., Garvie M. B. : Seasonal activity and frequency distributions of ticks (Acari: Ixodidae) infesting snowshoe hares in Nova Scotia, Canada. J. Med. Entomol., $1980,1 \%, 22-29$.

Cliffrord C., Flux J. E. C., Hoogstraal H. : Seasonal and regional abundance of ticks (Ixodoidea) on hares (Leporida) in Kenia. J. Med. Ent., 1976, 13, 40-47.

Daniel M., Cerny V., Dusbabek F., Honzakova E., Olejnicek J. : Influence of the microclimate on the life cycle of common tick Ixodes ricinus (L.) in an open area in comparison with forest habitats. Folia Parasit. (Praha), 1974, 24, 149-160.

Daniel M., Szymansky S., Cerny V., Dusbabek F., Honzakova E., Olejnicek J. : A comparaison of developmental dynamics of Dermacentor reticulatus (Fabr.) of different geographic origins and their affection by different microclimate. Folia Parasit. (Praha), 1980, 27, 63-69.

Eddy G. W. : Notes on the seasonal history of the rabbit tick, Haemaphysalis leporispalustris in Oklahoma. Proc. Entomol. Soc. Wash., 1942, 44, 145-149.

Edwards E. E., Arthur D. R. : The seasonal activity of the tick Ixodes ricinus L. in Wales. Parasitology, 1947, 38, 72-85.

Gilot B., Pautou G., Moncada E., Ain G. : Première contribution à l'étude écologique d'Ixodes ricinus (Linne, 1758) (Acarina, Ixodidae) dans le Sud-Est de la France. Acta Trop., 1975, $32,355-381$.

Gilot B., Perez C. : Individualisation et caractérisation de deux Ixodes actuellement confondus : I. festai Rondelli, 1926, I. ventalloi Gil Collado, 1936 (Acarina, Ixodoidea). Rev. Suisse Zool., 1978, 85, 143-149.

Gilot B., Rogers P. M. : Seasonal abundance of ticks on rabbits in the Camargue, Southern France. Proc. First Inter. Conf. Lagomorphs, Guelph, Canada, 1979, 667.

Gilot B., Rogers P., Lachet B. : Données biologiques et écologiques des tiques de Lagomorphes (et plus spécialement celles du lapin de garenne, Oryctolagus cuniculus L.) dans les Alpes françaises et leur avant-pays. Acarologia, 1985, 26, 335-354.

Green R. G., Ecans C. A., Larson C. L. : A ten-year population study of the rabbit tick Haemaphysalis leporispalustris. Am. J. Hyg., 1943, 38, 260-281.

Honzakova V., Cerny V., Daniel M., Dusbabek F. : Development of the tick Ixodes laguri Ol. in the nest of the european suslik Citellus citellus (L.). Folia Parasit. (Praha), 1980, $27,71-75$.

Hoogstran H., Morel P. C. : Haemaphysalis hispanica Gil Collado, 1936, a parasite of the european rabbit, redescription of adults and description of immatures stages (Ixodoidea: Ixodidae). J. Parasitol., 1970, 56, 813-822.

Lees A. D., Milnow A. : The seasonal and diurnal activities of individual Sheeps ticks (Ixodes ricinus L.). Parasitology, 1951, 41, 189-208.

Legendre L., Legendre P. : Écologie numérique. Tome 2. La structure des données écologiques. Masson/Presses de l'Université du Québec, 1984, $335 \mathrm{p}$.

Linduska J. P. : The ferret as an aid to winter rabbit studies. J. Wildl. Manage., 1947, 11, 523525.

Main A. J., Carey A. J., Carey M. G., Goodwin R. H.: Immature Ixodes dammini (Acari: Ixodidae) on small animals in Conneticut, USA. J. Med. Ent., 1982, 19, 655-664.

MACLEOD J.: Ixodes ricinus in relation to its physical environment. IV. An analysis of the ecological complexes controlling distribution and activities. Parasitology, 1936, 28, 295-319. 
Marquez F. J., Fernandez-Valdivia J. : Evolución anual de las poblaciones de Ixodoideos parásitos del Conejo de Monte (Oryctolagus cuniculus), en una formación de encinar mesomediterráneo de la provincia de Granada. Resumenes de Comunicaciones del V Congr. Nacional Parasitol., Salamanca, 1987, 115-116.

Marquez F. J., Jimenez-Millan F. : Estudio comparativo del gnatosoma de los Ixódidos del Conejo. III Congr. Iber. Entomol., Granada, 1988a. Sous presse.

Marquez F. J., Jimenez-Millan F. : Hacia un modelo del ciclo biológico de Rhipicephalus pusillus Gil Collado, 1938 (Acarina, Ixodidae). Resultados del muestreo anual de 1986. Rev. Ibér. Parasitol., 1988b, 48, 433-443.

Mcgowan M. J., Camin J. H., Macnew R. W. : Field study of the relationship between skinsensitibizing antibody production in the cottontail rabbit, Sylvilagus floridanus, and infestation by the rabbit tick, Haemaphysalis leporispalustris (Acari: Ixodidae). J. Parasitol., 1979, 65, 692-699.

Mermod C., Aeschlimann A., Graf J. F. : Écologie et éthologie d'Ixodes ricinus Linné, 1758 en Suisse (Acarina, Ixodoidea). Première note : Fluctuations numériques. Acarologia, 1973, 15, 197-205.

Mermod C., Aeschlimann A., Graf J. F. : Écologie et éthologie d’Ixodes ricinus L. en Suisse. Deuxième note : Comparaison des populations 1972 et 1973. Acarologia, 1974, 16, 612-620.

Mermod C., Aeschlimann A., Graf J. F. : Écologie et éthologie d'Ixodes ricinus L. en Suisse. Quatrième note : Comparaison de deux populations d'altitude différente. Acarologia, 1975, $17,442-451$.

Milne A. : The Ecology of the sheep tick Ixodes ricinus l. Some further aspects of activity, seasonal and diurnal. Parasitology, 1947, 38, 27-33.

Morel P. C., VAssiliades G. : Les Rhipicephalus du groupe sanguineus, espèces africaines (Acariens: Ixodoidea). Rev. Elev. Med. Vét. Pays Trop., 1962, 15, 343-386.

Norval R. A. I. : Ecology of the tick Amblyomma hebraeum Koch in the Eastern Cape province of South Africa. I. Distribution and seasonal activity. J. Parasitol., 1977, 63, 734-739.

Palacios F. : On the taxonomic status of the genus Lepus in Spain. Acta Zool. Fennica, 1983, $174,27-30$.

Piesman J., Spielman A. : Host associations and seasonal abondance of immature Ixodes dammini in Southern Massachusetts. Ann. Ent. Soc. America, 1979, $72,823-832$.

RANDOLPH S. E. : Seasonal dynamics of a host-parasite system: Ixodes trianguliceps (Acarina: Ixodidae) and its small mammals hosts. J. Anim. Ecol., 1975, 44, 425-429.

RANDolph S. E. : Patterns of distribution of the tick Ixodes trianguliceps Birula on its hosts J. Anim. Ecol., 1975, 44, 451-474.

RANDolph S. E. : Population regulation in ticks: the role of acquired resistance in natural and unnatural hosts. Parasitology, 1979, $79,141-156$.

Rechav Y. : Dynamics of tick populations (Acari: Ixodidae) in the Eastern Cape Province of South Africa. J. Med. Ent., 1982, 19, 679-700.

Rodriguez J. A. : Estudio de ectoparásitos de Lepóridos en la provincia de Ciudad Real. Tesis Doctoral, 1980. Univ. Complutense. Madrid. 383 p.

Rodriguez J. A., Zapatero L. M., Sanchez-Covisa A. : Evolución de ectoparásitos de lepóridos en el transcurso del año. II Congreso Nacional de Parasitologia, León, 1979.

Rodriguez J. A., Zapatero L. M., SAnchez-Covisa A. : Variación estacional de Ixodidae y Siphonaptera de Lepóridos en la provincia de Ciudad Real. Rev. Iber. Parasitol., 1981, 41, 527-538.

Schulze T. L., Gowen G. S., Lakat M. F., Parkin W. E., Shisler J. K. : Seasonal abundance and hosts of Ixodes dammini (Acari: Ixodidae) and other ixodid ticks from an endemic Lyme disease focus in New Jersey, USA. J. Med. Entomol., 1986, 23, 105-109.

Soriguer R. C. : Biología, ecología y productividad del conejo, Oryctolagus cuniculus (L.), en e] Sur de España. Tesis Doctoral, 1979. Uni. Sevilla.

Soriguer R. C. : El conejo, Oryctolagus cuniculus (L.), en Andalucía Occidental: Parámetros corporales y curva de crecimiento. Doñana, Acta Vertebrata, 1980a, \%, 83-90.

Soriguen R. C. : Ciclo anual de parasitismo por pulgas y garrapatas en el Conejo de Campo (Oryctolagus cuniculus L.) en Andalucía Occidental, España. Rev. Iber. Parasit., 1980b, $40,539-550$.

Soriguer R. C. : Estructura de sexos y edades en una población de conejos (Oryctolagus cuniculus L.) de Andalucía Occidental. Doñana, Acta Vertebrata, 1981, 8, 225-236.

Soriguer R. C. : Biología y dinámica de una población de Conejos (Oryctolagus cuniculus, L) en Andalucía Occidental. Doñana Acta Vertebrata, 1981, 8, Num. Esp., 1-379.

Willadsen P. : Immunity to ticks. Adv. Parasitol., 1980, 18, 293-313.

Wilson M. L., Spielman A. : Seasonal activity of immature Ixodes dammini (Acari: Ixodidae). J. Med. Entomol., 1985, 22, 408-414. 